\title{
Huaier polysaccharide induces apoptosis in hepatocellular carcinoma cells through p38 MAPK
}

\author{
HAIDONG BAO ${ }^{1 *}$, PENG LIU $^{1 *}$, KE JIANG $^{2}$, XIANBIN ZHANG ${ }^{1}$, \\ LONG XIE $^{1}$, ZHONGYU WANG ${ }^{1}$ and PENG GONG ${ }^{1}$ \\ ${ }^{1}$ Department of Hepatobiliary Surgery, The First Affiliated Hospital of Dalian Medical University, Dalian, Liaoning 116011; \\ ${ }^{2}$ Institute of Cancer Stem Cell, Dalian Medical University Cancer Center, Dalian, Liaoning 116044, P.R. China
}

Received March 31, 2015; Accepted May 13, 2016

DOI: $10.3892 / \mathrm{ol} .2016 .4686$

\begin{abstract}
The underlying mechanism of the antitumor activity of Huaier polysaccharide (HP) remains to be explored. The present study aimed to investigate the inhibitory effect of HP on hepatocellular carcinoma (HCC) cells, and to explore the possible mechanisms of its anticancer effect. Cell viability was measured by 3-(4,5-dimethylthiazol-2-yl)-2,5-diphenyltetrazolium bromide assay, while apoptotic nuclear changes were observed using Hoechst 33258 staining. The distribution of cell cycle and apoptosis were analyzed by flow cytometry, and western blotting was used to test the apoptotic pathways. Apoptosis and mitogen-activated protein kinase (MAPK) inhibitors were used to investigate the mechanism of apoptosis. HP triggered cell cycle arrest and apoptosis in HepG2 and Huh7 cells. Both the extrinsic and intrinsic apoptotic pathways were activated after HP treatment. Furthermore, HP enhanced the three major MAPK pathways (extracellular signal-regulated kinase, c-Jun N-terminal kinase and p38 MAPK) and inhibited the AKT/mechanistic target of rapamycin signaling pathway in HCC cells. Notably, the inactivation of p38 MAPK impaired the HP-induced cell death. HP exerted its antitumor effect on HCC cells through the regulation of the expression of the apoptosis-related proteins B-cell lymphoma (Bcl)-2, Bcl-2-associated $\mathrm{X}$ protein and survivin. The present study provides evidence that HP induces apoptosis in HCC cells and demonstrated the role of p38 MAPK in HP-triggered cancer cell death.
\end{abstract}

Correspondence to: Professor Peng Gong, Department of Hepatobiliary Surgery, The First Affiliated Hospital of Dalian Medical University, 222 Zhongshan Road, Dalian, Liaoning 116011, P.R. China

E-mail: gongpengdalian@163.com

${ }^{*}$ Contributed equally

Key words: apoptosis, hepatocellular carcinoma, mitogen-activated protein kinase, p38 MAPK, Huaier polysaccharide

\section{Introduction}

Liver cancer, particularly hepatocellular carcinoma (HCC), is one of the most common human malignancies with poor long-term survival rates (1-3). Although liver transplantation, hepatectomy and local therapy are potentially curative therapies in the early stages of HCC $(4,5)$, the majority of patients, who are usually diagnosed at an advanced stage, must rely mainly on traditional chemotherapies $(6,7)$. Currently, there is no proven effective conventional systemic chemotherapy for patients with advanced HCC (7). Therefore, novel therapeutic agents with high efficacy are urgently required for the clinical treatment of advanced HCC.

Trametes robiniophila Murr (Huaier) is a type of fungus that exists in China, and previous chemical analyses revealed that Huaier consists mainly of polysaccharide (8). Recent studies have noticed that Huaier polysaccharide (HP) exerts a pro-apoptotic effect on the cells of a variety of human cancers, including breast cancer $(9,10)$, hepatocarcinoma (11-14), lung adenocarcinoma (15) and ovarian cancer (16). In addition, Huaier and HP suppress cancer cell metastasis and motility $(12,16,17)$, exhibit anti-angiogenic activity and enhance the host immune system function $(11,14,18)$. Together, these data indicate that HP exhibits promising results against cancer in pre-clinical trials.

The use of Huaier has been approved by the Chinese Food and Drug Administration for the clinical treatment of patients with malignant tumors (China Food and Drug Administration approval number, Z20000109; http://app1.sfda.gov.cn/datasearch/face3/base.jsp). Although several studies indicated that HP induces apoptosis in HCC cells via different signaling pathways $(13,19)$, the detailed mechanism by which this drug inhibits HCC cell growth remains to be explored.

Mitogen-activated protein kinase (MAPK) participate in the regulation of cell proliferation, differentiation, cellular stress responses and apoptosis $(20,21)$. The activation of the three major MAPK pathways [extracellular signal-regulated kinase (ERK)1/2, c-Jun N-terminal kinase (JNK) and p38 MAPK], has been implicated in the activity of numerous chemotherapy and genotoxic drugs (22). Therefore, HP may participate and regulate proliferation and apoptosis of HCC cells through the MAPK signaling pathway.

The present study focused on the inhibitory effect of HP on both HepG2 and Huh7 HCC cells, and explored the possible 
mechanisms of its anticancer effect. Furthermore, the critical role of MAPK in the regulation of these processes was investigated.

\section{Materials and methods}

Antibodies and reagents. Polyclonal rabbit caspase-3 (catalog no. 9662S), monoclonal mouse caspase-8 (catalog no. 9746), polyclonal rabbit caspase-9 (catalog no. 9502), monoclonal rabbit phosphorylated (p)-p38 (catalog no. 9215S), polyclonal rabbit p-AKT (catalog no. 9271S), polyclonal rabbit total JNK (catalog no. 9252), polyclonal rabbit total p38 (catalog no. 9212), polyclonal rabbit total AKT (catalog no. 9272S), monoclonal rabbit B-cell lymphoma (Bcl)-2 (catalog no. 2870S), polyclonal rabbit Bcl-2-associated X protein (Bax; catalog no. 2772S), polyclonal rabbit Bcl-extra large (xL) (catalog no. 2762S), monoclonal rabbit myeloid cell leukemia-1 (Mcl-1; 5453S), monoclonal rabbit Bcl-2-like 11 (also known as Bim; catalog no. 2933S), polyclonal rabbit p53 (catalog no. 9282) and monoclonal mouse survivin (catalog no. 2802S) antibodies were purchased from Cell Signaling Technology, Inc. (Danvers, MA, USA). The dilution ratio of all of these antibodies was 1:1,000.

Polyclonal rabbit Anti-poly (ADP-ribose) polymerase (PARP) p85 fragment (catalog no. G734A), anti-ERK (catalog no. V114A), anti-p-JNK (V793B) and anti-active ERK1/2 (catalog no. V803A) antibodies were obtained from Promega Corporation (Madison, WI, USA). The dilution ratio of all of these antibodies was 1:4,000.

Polyclonal rabbit cyclin D1 (catalog no. sc753) and monoclonal mouse cyclin-dependent kinase 2 (CDK2; catalog no. sc6248) antibodies were obtained from Santa Cruz Biotechnology, Inc. (Dallas, TX, USA). The dilution ratio of these antibodies was 1:500.

Polyclonal rabbit glyceraldehyde 3-phosphate dehydrogenase antibody (10494-1-AP; 1:8,000) was purchased from Proteintech Group (Rosemont, IL, USA). Polyclonal rabbit p70S6 kinase antibody (catalog no. ABS431; 1:1,000 dilution) was purchased from EMD Millipore.

Specific inhibitors of MAPK kinase (MEK) (PD98059) (catalog no. 513000-5MGCN), JNK (SP600125) (catalog no. 420119-5MGCN) and p38 (SB203580) (catalog no. 559389-1MGCN) were purchased from Calbiochem (EMD Millipore, Billerica, MA, USA). 3-(4,5-dimethylthiazol-2-yl)-2,5-diphenyltetrazolium bromide (MTT) was obtained from Amresco, LLC (Solon, OH, USA). The pan-caspase peptide inhibitor Z-VAD-FMK was purchased from Promega Corporation and prepared in dimethyl sulfoxide (DMSO). HP was donated by Qidong Gaitianli Pharmaceutical Co., Ltd. (Jiangsu, China).

Cell culture. The two types of HCC lines (HepG2 and Huh7) were obtained from the American Type Culture Collection (Manassas, VA, USA) and were routinely maintained in Dulbecco's modified Eagle medium supplemented with $10 \%$ fetal bovine serum, $100 \mathrm{U} / \mathrm{ml}$ penicillin and $100 \mu \mathrm{g} / \mathrm{ml}$ streptomycin. The cells were incubated at $37^{\circ} \mathrm{C}$ in a $5 \% \mathrm{CO}_{2}$ air incubator.

Measurements of cell viability by MTT assay. Cells were seeded in 96-well plates. After incubation overnight, the medium was replaced with different concentrated solutions of HP and incubated for 24, 48 and $72 \mathrm{~h}$. Next, $10 \mu \mathrm{l}$ MTT was added to each well and the cells were incubated for $4 \mathrm{~h}$. Subsequently, the solutions in each well were carefully replaced by $100 \mu \mathrm{l}$ DMSO for $10 \mathrm{~min}$. The absorbance values were read with a microplate reader (PerkinElmer, Inc., Waltham, MA, USA) at $490 \mathrm{~nm}$ wavelength. Each experiment was conducted in triplicate.

Effect of HP on cell morphology. Cells were treated with $\mathrm{HP}$ at a concentration of $100 \mu \mathrm{g} / \mathrm{ml}$ for 24,48 and $72 \mathrm{~h}$. The morphological changes of the treated and untreated cells were observed at 24, 48 and $72 \mathrm{~h}$ under a light microscope (Olympus Corporation, Tokyo, Japan), and photomicrographs were captured with a digital camera (Olympus Corporation).

Cell cycle analysis. A total of $3 \times 10^{5}$ cells/well were seeded in 6-well plates and incubated overnight. After starvation with serum-free medium for $12 \mathrm{~h}$, the cells were incubated for $24 \mathrm{~h}$ in a HP-containing solution (50 or $100 \mu \mathrm{g} / \mathrm{ml}$ ). Next, the cells were trypsinized and fixed in cold ethanol. Subsequently, the cells were centrifuged at $168 \mathrm{x}$ g for $5 \mathrm{~min}$ and washed twice with phosphate-buffered saline (PBS). Next, RNase (KeyGen BioTech, Corporation Ltd., Nanjing, China), 2\% Triton X-100 and PBS were added to the cells per Eppendorf tube, followed by the addition of propidium iodide (PI) to stain the DNA of the cells. The DNA contents were quantified using an Accuri C6 flow cytometer (BD Biosciences, Franklin Lakes, NJ, USA).

PI-annexin V staining analysis. Cell apoptosis rates were determined by a flow cytometric analysis. In brief, HepG2 and Huh7 cells were treated with HP (100 $\mu \mathrm{g} / \mathrm{ml})$ for 0,12 , 24 and $48 \mathrm{~h}$. Then, the cells were harvested and resuspended in binding buffer (KeyGen BioTech, Corporation Ltd.). Aliquots of $5 \mu \mathrm{l}$ annexin $\mathrm{V}$-fluorescein isothiocyanate and $5 \mu \mathrm{l}$ PI were added to the cells, and the samples were then analyzed by flow cytometry on an Accuri C6. Cell apoptosis rates were determined in three independent experiments.

Hoechst 33258 staining. Apoptotic nuclear changes were observed using Hoechst 33258 (Sigma-Aldrich, St. Louis, MO, USA) staining. Cells were treated with HP $(100 \mu \mathrm{g} / \mathrm{ml})$ for different time periods. The treated cells were fixed with $4 \%$ paraformaldehyde and stained with Hoechst $33258(5 \mu \mathrm{g} / \mathrm{ml})$. The apoptotic nuclear changes of the cells were examined with a fluorescence microscope equipped with a digital camera (Olympus Corporation).

Immunoblot analysis. Cells were treated with HP at a concentration of $100 \mu \mathrm{g} / \mathrm{ml}$ at different time points $(0,4,8,12,24$ and $48 \mathrm{~h}$ ). The cells were lysed in a lysis buffer in the presence of protease inhibitors. Equal amounts of protein samples were separated by $7.5-15 \%$ sodium dodecyl sulfate-polyacrylamide gel electrophoresis and transferred onto nitrocellulose membranes. After blocking with $5 \%$ non-fat milk, the membranes were incubated overnight at $4{ }^{\circ} \mathrm{C}$ with the primary antibodies. On the following day, the membranes were washed and incubated with the goat anti-mouse immunoglobulin (Ig)G-horseradish peroxidase (HRP) (catalog no. sc-2005; 1:5,000 dilution; Santa Cruz Biotechnology Inc.) and goat anti-rabbit immunoglobulin (Ig)G-HRP (catalog no. sc-2030; 1:5,000 dilution; Santa Cruz Biotechnology, Inc.) secondary antibodies for $1 \mathrm{~h}$ at room temperature. The protein bands were detected using enhanced 
A

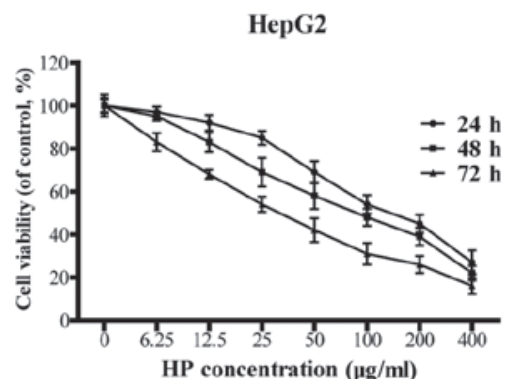

Huh7

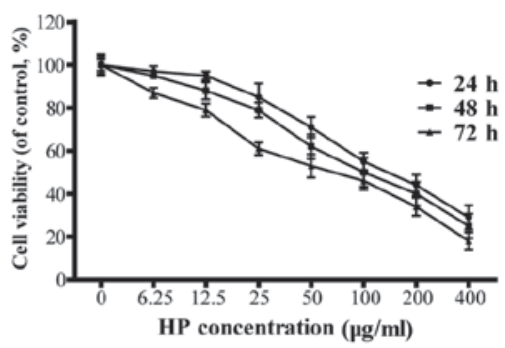

B
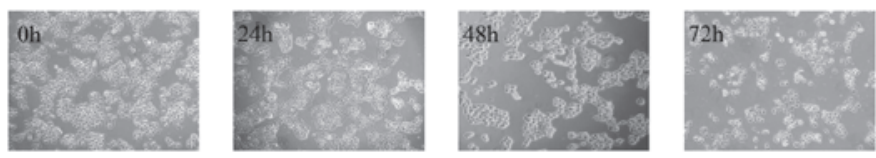

HepG2
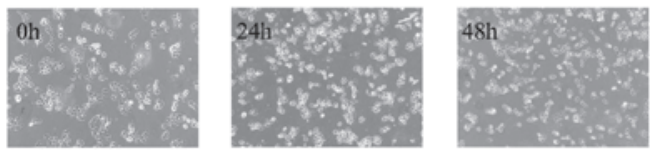

C

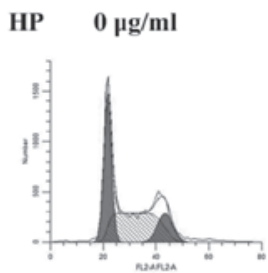

Huh7
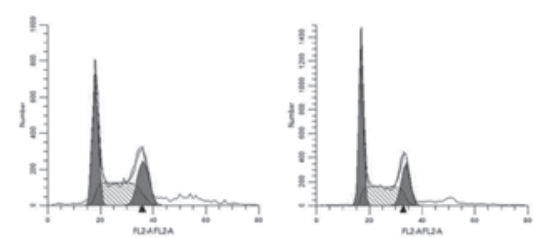
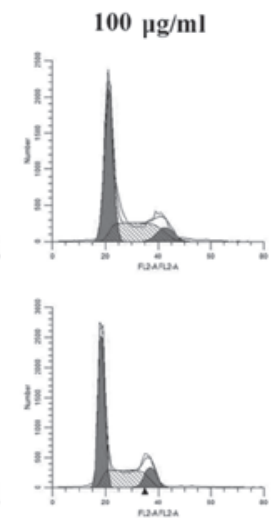

E

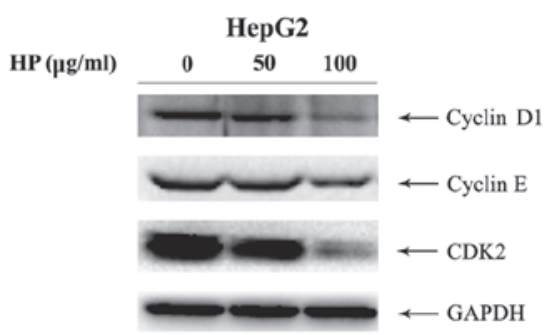

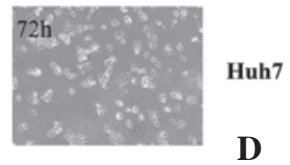

D

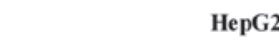

$\mathrm{G} 0 / \mathrm{Gl}$

$\otimes \mathrm{S}$

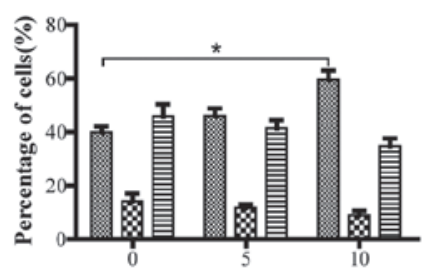

E $\mathrm{G} 2 / \mathrm{M}$

HP concentration $(\mu \mathrm{g} / \mathrm{ml})$

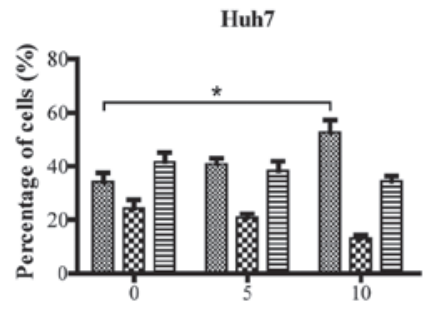

HP concentration $(\mu \mathrm{g} / \mathrm{ml})$

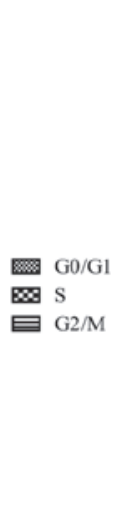

$\mathbf{S} \mathrm{S}$

Figure 1. HP inhibits cell viability and causes cell morphology changes in HepG2 and Huh7 cells. (A) Cells were treated with HP for 24,48 and 72 h. The cell viability of both HepG2 and Huh7 cells decreased in a dose- and time-dependent manner. The data are presented as the mean \pm SEM from three independent experiments. (B) Contrast in cell morphology. Changes in HepG2 and Huh7 cells were observed following the addition of $\mathrm{HP}(100 \mu \mathrm{g} / \mathrm{ml})$ at $0,24 \mathrm{and} 48 \mathrm{~h}$ after the treatment (magnification, x100). (C and D) HP induces cell cycle arrest in HepG2 and Huh7 cells upon treatment with 50 and $100 \mu \mathrm{g} / \mathrm{ml}$ for $24 \mathrm{~h}$. (C) The distribution of the cell cycle of HepG2 and Huh7 cells was detected by flow cytometry after staining with propidium iodide. (D) Quantification of the results of panel C. The data are presented as the mean \pm SEM from three independent experiments. " $\mathrm{P}=0.023$ for HepG2 and $\mathrm{P}=0.028$ for Huh7. (E) Detection of cyclin D1, cyclin E and cyclin-dependent kinase 2, which are biomarkers of G1 phase. The protein levels of glyceraldehyde 3-phosphate dehydrogenase were used as a loading control. All immunoblotting experiments were performed twice. CDK2, cyclin-dependent kinase 2; GAPDH, glyceraldehyde 3-phosphate dehydrogenase; HP, Huaier polysaccharide; SEM, standard error of the mean.

chemiluminescence (Pierce; Thermo Fisher Scientific, Inc, Waltham, MA, USA) and analyzed by Gel Doc XR+ (Bio-Rad Laboratories, Inc., Hercules, CA, USA).

Inhibitors treatment. For the apoptosis assay and the study of the detailed regulatory mechanism of apoptosis following HP treatment, inhibitors of apoptosis and MAPK were used.HepG2 and Huh7 cells were incubated with $50 \mu \mathrm{M} \mathrm{Z-VAD-FMK} \mathrm{or}$ MAPK inhibitors (PD98059, $20 \mu \mathrm{M}$; SP600125, $10 \mu \mathrm{M}$; and
SB203580, $10 \mu \mathrm{M}$ ) for $1 \mathrm{~h}$ prior to treatment with HP. Control cultures were treated with DMSO, which was used as a solvent for the peptide inhibitors. The treated cells were monitored by MTT assay, flow cytometry or immunoblot analysis for apoptosis.

Statistical analysis. All reported data are presented as the mean \pm standard error of the mean of three replicates. Student's $t$-test was used for determining the statistical difference 
A
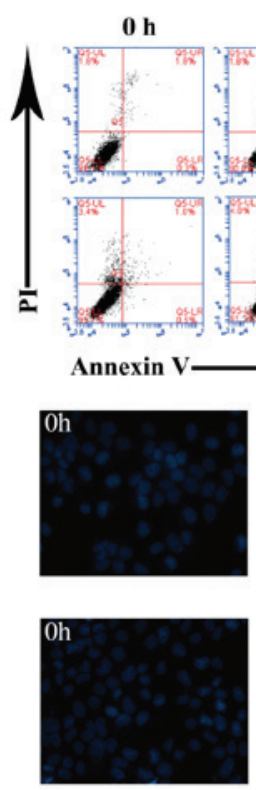

D

HepG2

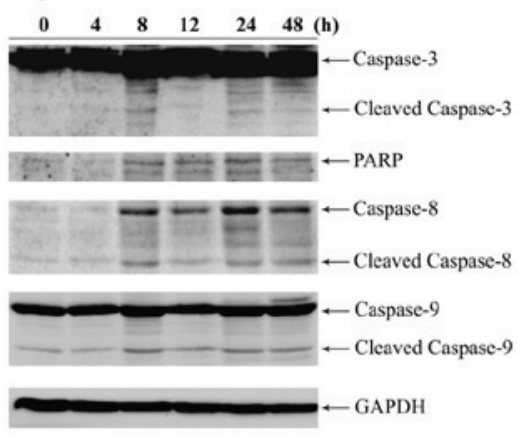

$\mathbf{E}$

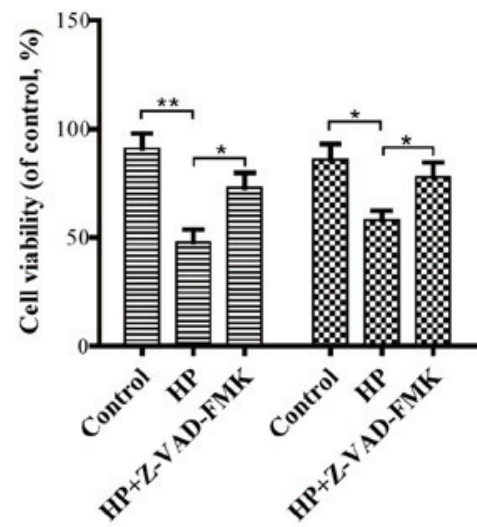

B
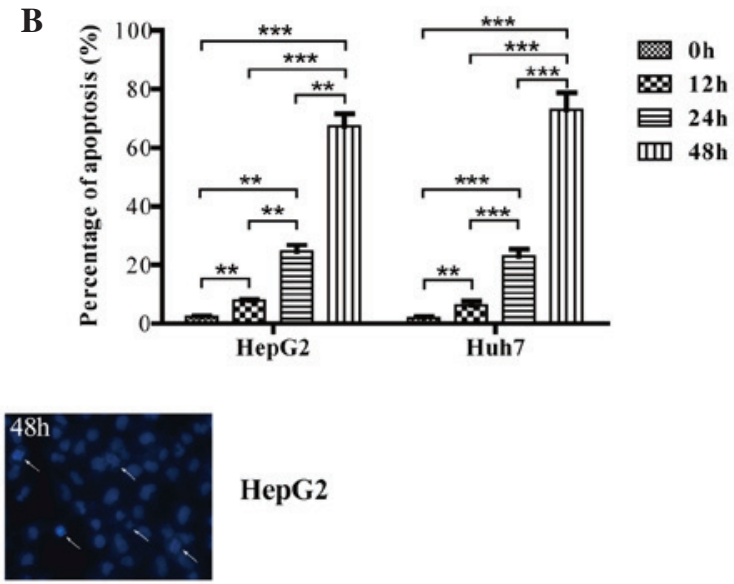

HepG2

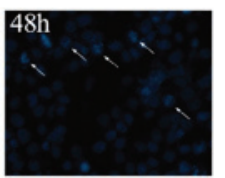

Huh7
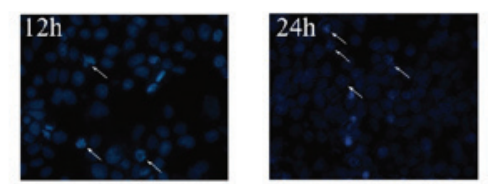

Huh7

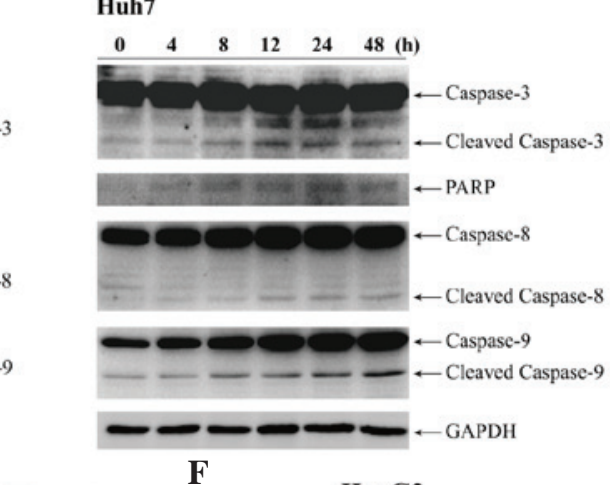

回 HepG2

$\mathbf{F}$

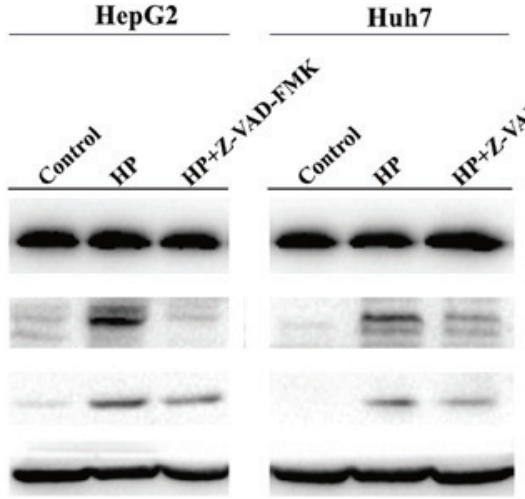

Caspase-3

Cleaved Caspase-3

Cleaved PARP

GAPDH

Figure 2. HP induces apoptosis in HepG2 and Huh7 cells in vitro. (A) Apoptosis was analyzed by flow cytometry, and the dot plots of cells treated with $100 \mu \mathrm{g} / \mathrm{ml} \mathrm{HP}$ for $0,12,24$ and $48 \mathrm{~h}$ were obtained. (B) Comparison of apoptosis rates at different times. The data are presented as the mean \pm SEM from three independent experiments. " $\mathrm{P}<0.05$ indicates a significant increase in the apoptosis rate in a time-dependent manner. ${ }^{* *} \mathrm{P}<0.01$ and ${ }^{* * * *} \mathrm{P}<0.001$. (C) The cells were treated with HP for 0, 12, 24 and $48 \mathrm{~h}$, stained with Hoechst 33258, and the cell morphology was analyzed by fluorescence microscopy (magnification, x200). (D) Detection of the activated forms of caspases-3, -8 and -9 and cleaved PARP. The protein levels of glyceraldehyde 3-phosphate dehydrogenase served as a loading control. The proteins were analyzed at the indicated time points, and all immunoblotting experiments were performed twice. (E) Measurements by 3-(4,5-dimethylthiazol-2-yl)-2,5-diphenyltetrazolium bromide assay of cell viability upon treatment with $100 \mu \mathrm{g} / \mathrm{ml} \mathrm{HP}$, with or without Z-VAD-FMK for $24 \mathrm{~h}$. Data are presented as the mean \pm SEM from three independent experiments. ${ }^{*} \mathrm{P}<0.05$ indicates a significant increase in the cell viability upon treatment with Z-VAD-FMK. (F) HepG2 and Huh7 cells were treated with HP $(100 \mu \mathrm{g} / \mathrm{ml})$, with or without $50 \mu \mathrm{M} \mathrm{Z-VAD-FMK} \mathrm{for} 24 \mathrm{~h}$. The cell lysates were prepared for immunoblotting to examine the expression of caspase-3 and PARP. UL, upper left; UR, upper right; LL, lower left; LR, lower right; PI, propidium iodide; PARP, poly (ADP-ribose) polymerase; GAPDH, glyceraldehyde 3-phosphate dehydrogenase; HP, Huaier polysaccharide; SEM, standard error of the mean.

between two groups, and one-way analysis of variance was performed for multiple comparisons. Statistical analysis was performed using SPSS 17.0 software (SPSS Inc., Chicago, IL, USA). $\mathrm{P}<0.05$ was considered to indicate a statistically significant difference.

\section{Results}

HP inhibits cell growth and induces cell cycle arrest in HCC cells. HepG2 and Huh7 cells (23), were treated with HP for the indicated time periods and concentrations, and subjected 
A

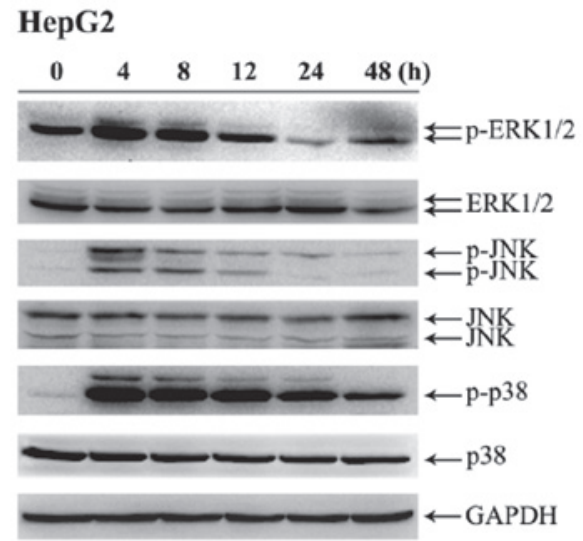

B

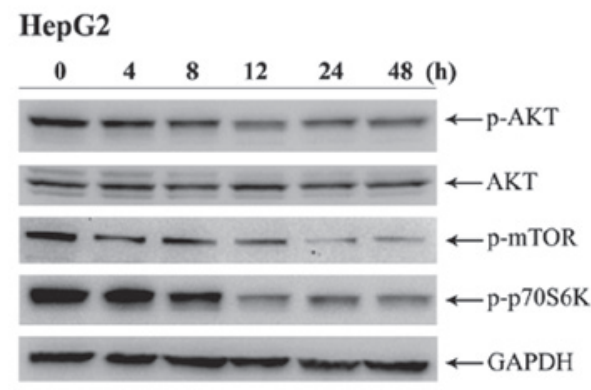

Huh7

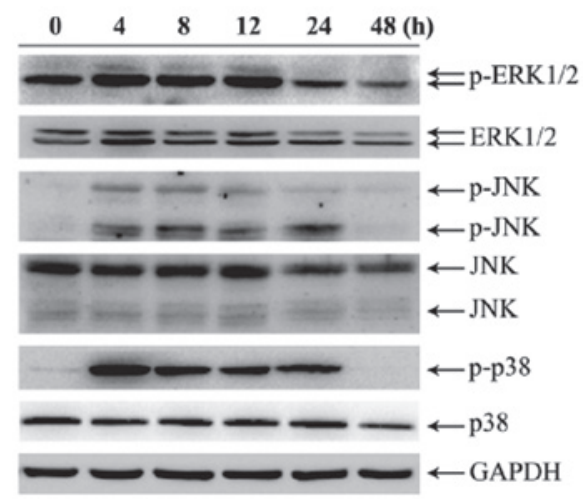

Huh7

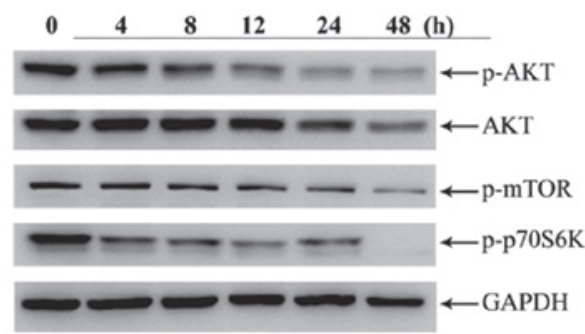

Figure 3. MAPK and AKT-mTOR signaling pathways in Huaier polysaccharide-treated HepG2 and Huh7 cells. (A) Activation of the MAPK signaling pathways was observed in both HepG2 and Huh7 cells using specific antibodies against activated extracellular signal-regulated kinase 1/2, c-Jun N-terminal kinase (JNK gene can produce 46- and 54-kDa proteins) and p38 (43 kDa protein). (B) Detection of the activated proteins in the AKT-mTOR signaling pathway. The cells were treated as described in Fig. 2, and the protein levels of glyceraldehyde 3-phosphate dehydrogenase were used as a loading control. All immunoblotting experiments were performed twice. p, phosphorylated; ERK, extracellular signal-regulated kinase; JNK, c-Jun N-terminal kinase; GAPDH, glyceraldehyde 3-phosphate dehydrogenase; mTOR, mechanistic target of rapamycin; MAPK, mitogen-activated protein kinase; S6K, S6 kinase.

to cell growth analysis. As shown in Fig. 1A, HP significantly suppressed the proliferation of HepG2 and Huh7 cells in a timeand dose-dependent manner. In addition, cell morphology examination revealed that the majority of the HP-treated cells presented the characteristics of shrinkage, irregularity, nuclear condensation and fragmentation compared with mock-treated cells (Fig. 1B), indicating cell damage induced by the HP treatment.

To investigate whether the HP-triggered inhibitory effect on $\mathrm{HCC}$ cell growth is due to cell cycle arrest, the cell cycle profiles were determined by flow cytometry. As illustrated in Fig. 1C and D, HP treatment triggered G0/G1 cell cycle arrest in both HepG2 and Huh7 cells. Cyclins D1 and E are the main proteins of cell cycle regulation in G1/S phase $(24,25)$. Fig. 1E depicts the dose-dependent decrease of the expression of cyclin D1, cyclin E and CDK2 in HepG2 cells following HP treatment, which was in line with the fluorescence-activated cell sorting data. Similar results were obtained in the HP-treated Huh7 cells, with the exception of cyclin E.

HP treatment triggers cell apoptosis in HCC cells. To investigate whether the HP-induced inhibitory effects on HCC cell proliferation are due to apoptosis, an annexin V/PI double staining assay was performed. As illustrated in Fig. 2A and B, HP exposure resulted in remarkable apoptosis in both HepG2 and Huh7 cells in a time-dependent manner. Hoechst 33258 staining revealed that apoptotic chromatin condensation in HepG2 and Huh7 cells was readily observed at all time points subsequent to HP exposure (Fig. 2C), whereas nearly no chromatin condensation was observed in the cells subjected to control treatment.

The activation of the death receptors (extrinsic) and mitochondria (intrinsic) apoptotic pathways was next examined. As shown in Fig. 2D, cleavage of caspase- 8 or caspase- 9 was observed in HepG2 and Huh7 cells, indicating that both the extrinsic and intrinsic apoptotic pathways were involved in the HP-induced apoptosis in HCC cells. Caspase-3 is the major effector of apoptosis, and PAPR is cleaved during the induction of apoptosis (26). Fig. 2D depicts the activated forms of caspase-3 and PARP cleavage that were detected in the HP-treated HCC cells.

To further confirm that HP-induced apoptosis in HepG2 and Huh7 cells is caspase-dependent, cells were pretreated with a broad-specificity caspase inhibitor (Z-VAD-FMK), and cell viability was analyzed by MTT assay and immunoblotting which revealed the expression of caspase-3 and PARP. As shown in Fig. 2E, cell viability was markedly recovered in cells treated with $50 \mu \mathrm{M} \mathrm{Z-VAD-FMK}$ for $24 \mathrm{~h}$ compared with those treated with HP alone. The expression of cleaved caspase- 3 and PARP was markedly inhibited compared with the HP-treated group (Fig. 2F). Therefore, these results indicated that caspase activity is required for HP-triggered apoptosis in HepG2 and Huh7 cells.

HP exposure activates MAPK pathways and downregulates AKT phosphorylation in HCC cells. To gain a further insight into the mechanism by which HP induces apoptosis, the phosphorylation levels of MAPK and AKT were investigated. As presented in Fig. 3A, $4 \mathrm{~h}$ after HP treatment, the 
A
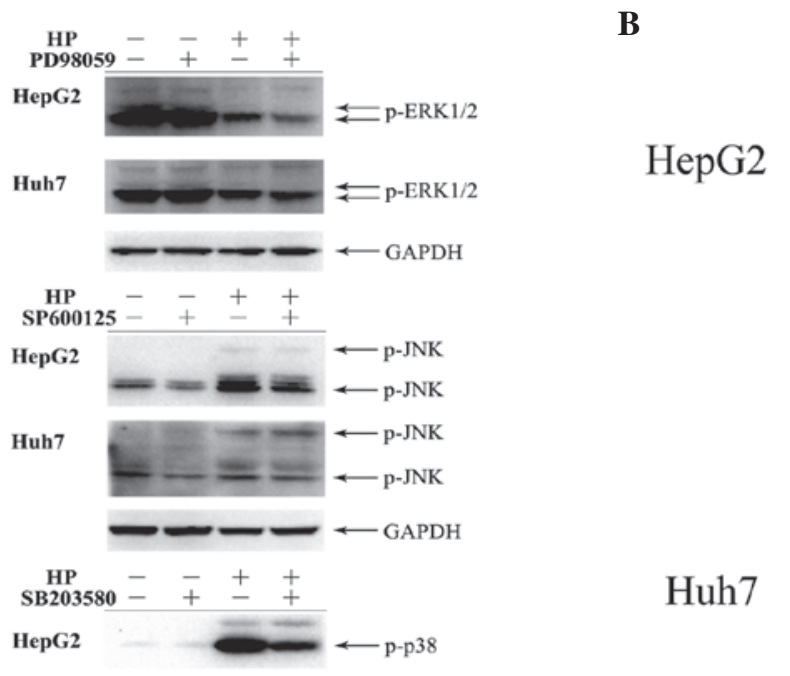

Huh7 $-\longleftarrow \mathrm{p}$-p38 (Short exposure)

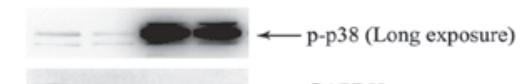

C

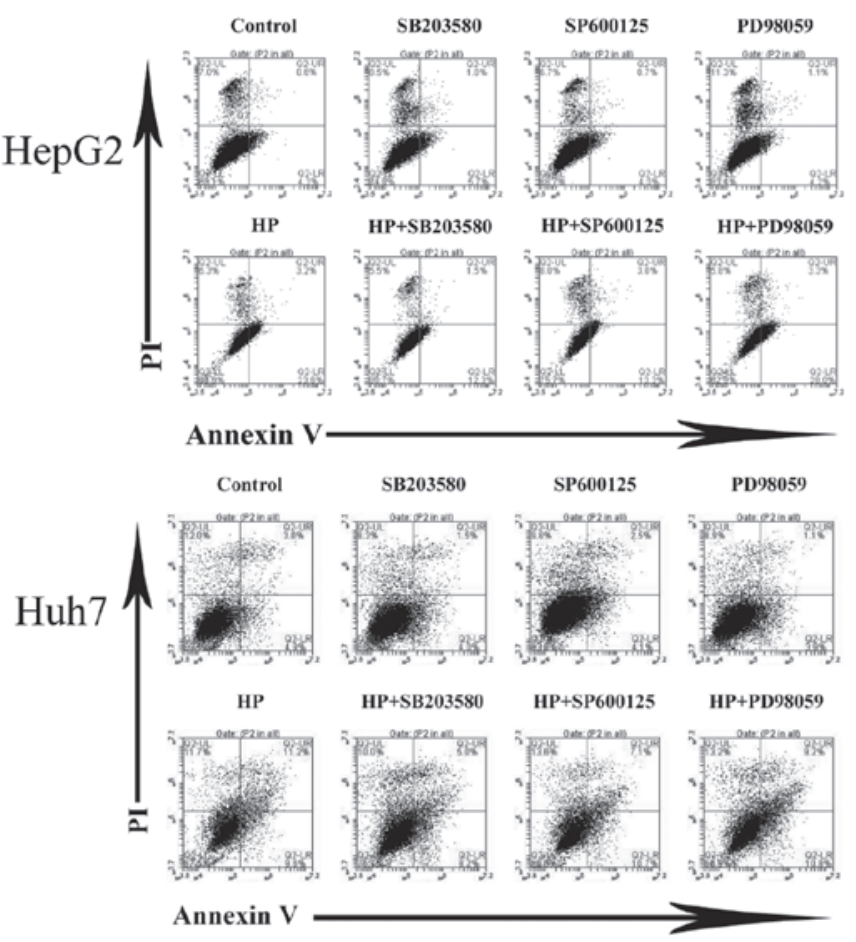

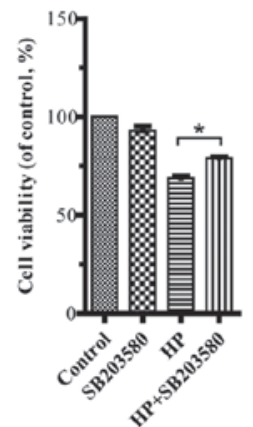
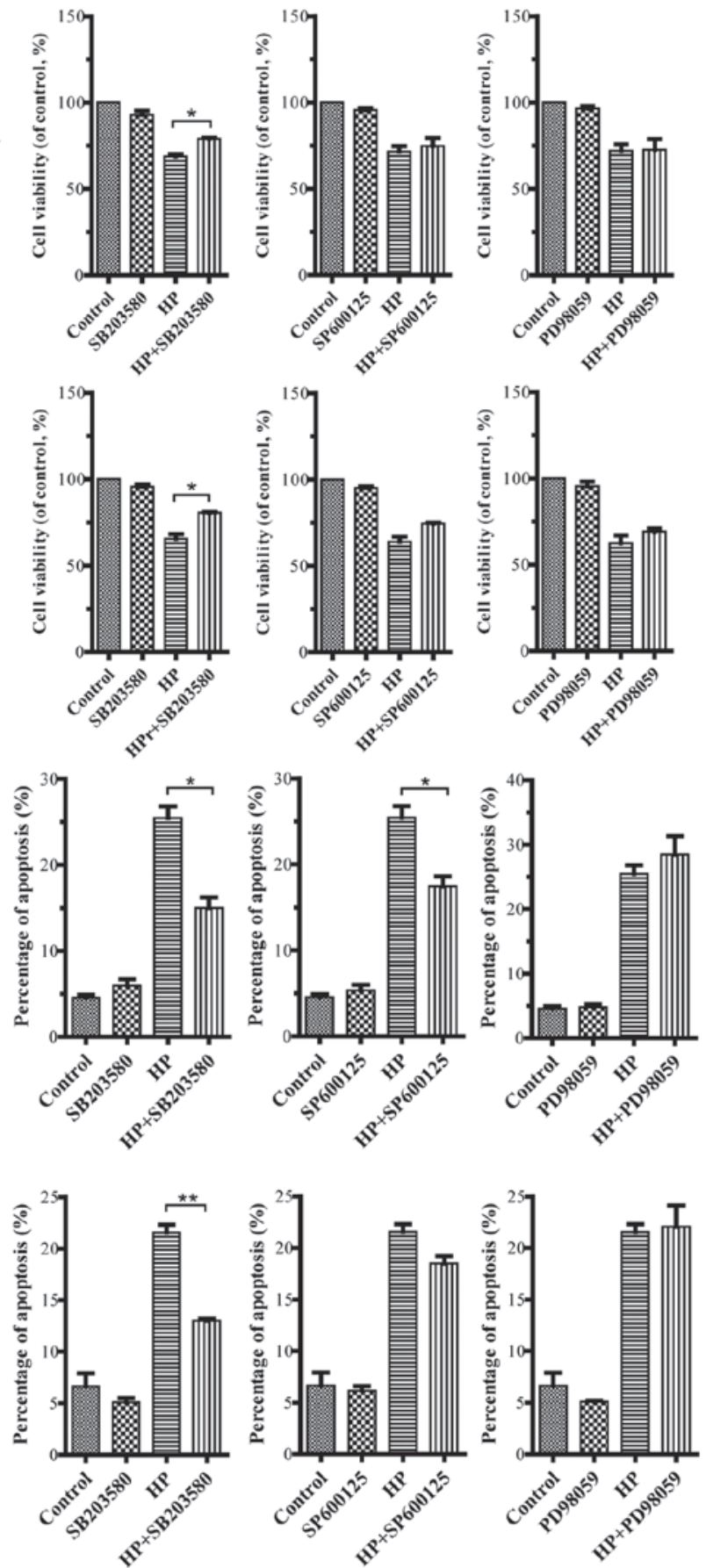

Figure 4. Effects of inhibiting the MAPK signaling pathway on cell viability and apoptosis. (A) HepG2 and Huh7 cells were pretreated with PD98059 (20 $\mu$ M), SP600125 $(10 \mu \mathrm{M})$ and SB203580 $(10 \mu \mathrm{M})$ for $24 \mathrm{~h}$. The cell lysates were prepared for immunoblotting to examine the inactivation of the target pathways. (B) Measurements by 3-(4,5-dimethylthiazol-2-yl)-2,5-diphenyltetrazolium bromide assay of cell viability upon treatment with $100 \mu \mathrm{g} / \mathrm{ml} \mathrm{HP}$, with or without MAPK inhibitors for $24 \mathrm{~h}$. The data are presented as the mean \pm SEM from three independent experiments. ${ }^{*} \mathrm{P}<0.05$ indicates a significant increase in cell viability following treatment with MAPK inhibitors. (C) Influence of the MAPK signaling pathway on apoptosis. Cell apoptosis was analyzed by flow cytometry upon treatment with $100 \mu \mathrm{g} / \mathrm{ml} \mathrm{HP}$, with or without MAPK inhibitors for $24 \mathrm{~h}$. The statistical results are presented in a bar chart. The data correspond to the mean \pm SEM from three independent experiments. ${ }^{*} \mathrm{P}<0.05$ and ${ }^{* *} \mathrm{P}<0.01$ indicate a significant decrease in the apoptosis rate following treatment with MAPK inhibitors. UL, upper left; UR, upper right; LL, lower left; LR, lower right; PI, propidium iodide; HP, Huaier polysaccharide; p, phosphorylated; ERK, extracellular signal-regulated kinase; JNK, c-Jun N-terminal kinase; GAPDH, glyceraldehyde 3-phosphate dehydrogenase; MAPK, mitogen-activated protein kinase; SEM, standard error of the mean.

phosphorylation levels of ERK1/2, JNK and p38 MAPK in HepG2 and Huh7 cells were markedly increased. Notably, p38 MAPK was robustly activated in the time interval from 4 to $24 \mathrm{~h}$ in both cell lines (Fig. 3A). On the contrary, the HP treatment attenuated the phosphorylation levels of both AKT and mechanistic target of rapamycin (mTOR) in HepG2 and Huh7 cells compared with their initial levels (Fig. 3B).
Pharmacological inhibition of p38 MAPK attenuates the HP-induced apoptosis in both HepG2 and Huh7 cells. The specific inhibitors PD98059, SP600125 and SB203580, which target MEK, JNK and p38 MAPK, respectively, were used to treat the cells prior to HP exposure. The effective concentrations of these inhibitors were tested in a dose-response assay for each inhibitor to prevent cytotoxicity (data not shown). As 
A

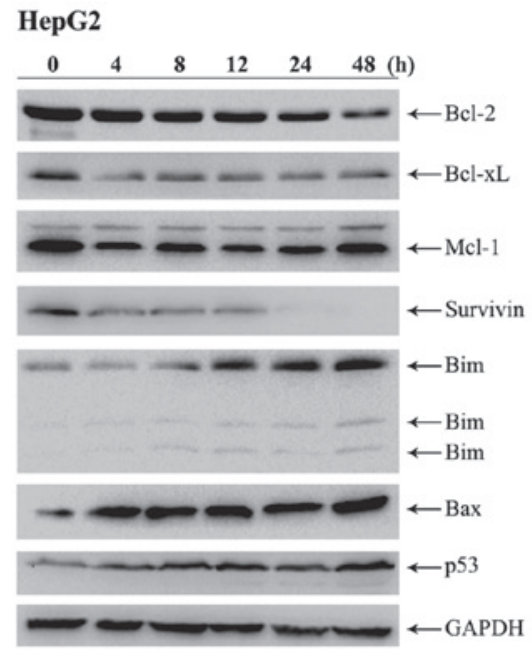

Huh7

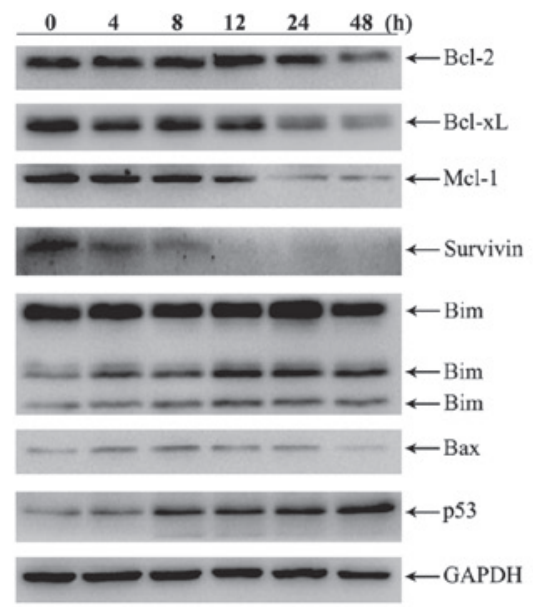

B

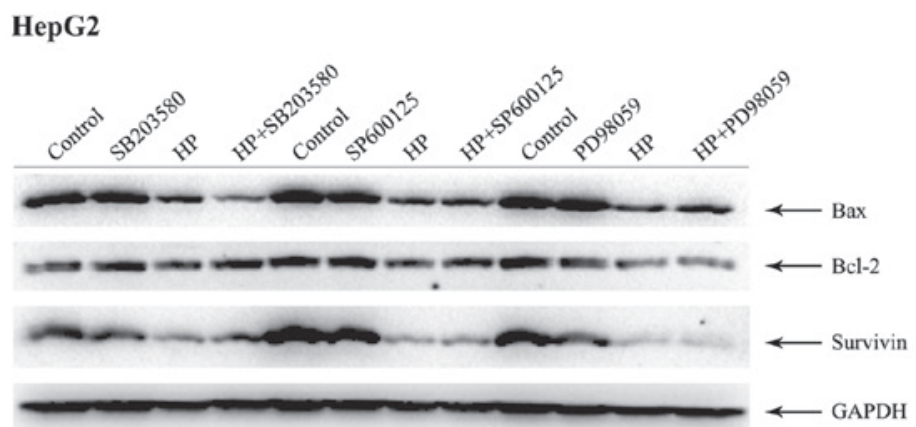

C

Huh7

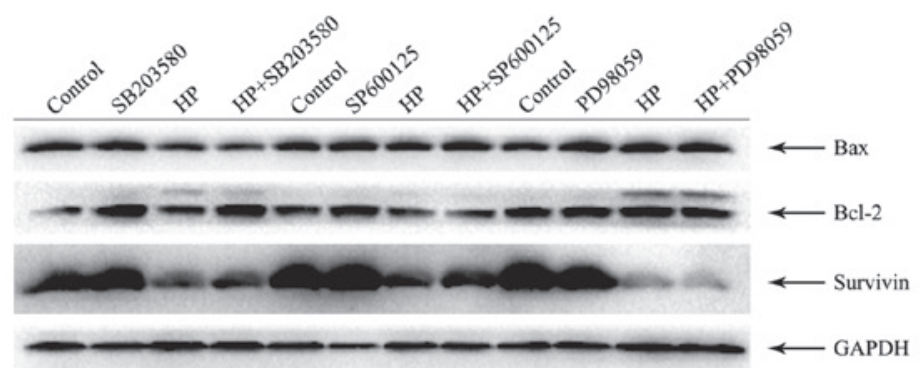

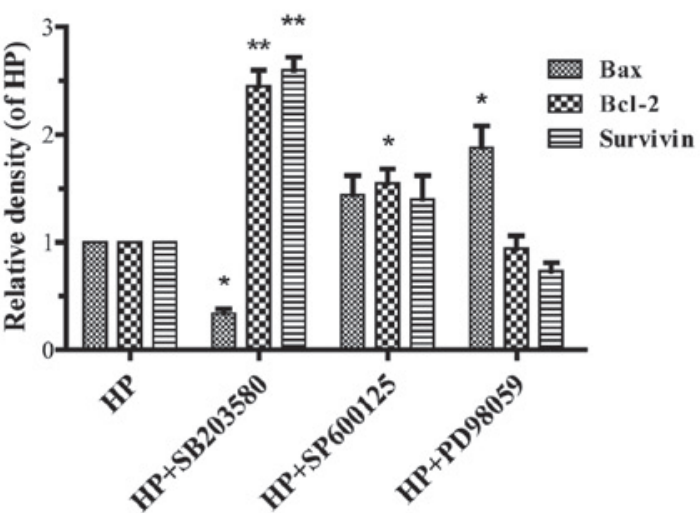

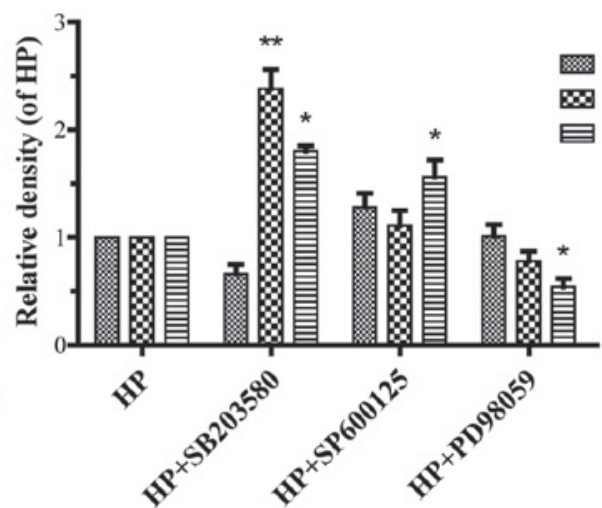

Figure 5. Effects of HP treatment and inhibition of the MAPK signaling pathway on the expression of AKT and mitochondrial pathway proteins. (A) Cells were treated with $\mathrm{HP}(100 \mu \mathrm{g} / \mathrm{ml})$ for $0,4,8,12,24$ and $48 \mathrm{~h}$. The different expression of the B-cell lymphoma-2 family proteins indicated that the mitochondrial pathway was affected after the treatment with HP. The expression of GAPDH was used as an internal control. All immunoblotting experiments were performed twice. (B and C) Effects of inhibiting the MAPK signaling pathway on the mitochondrial pathway proteins in (B) HepG2 and (C) Huh7 cells. HP treatment at a concentration of $100 \mu \mathrm{g} / \mathrm{ml}$ for $24 \mathrm{~h}$, with or without MAPK inhibitors (PD98059, $20 \mu \mathrm{M}$; SP600125, $10 \mu \mathrm{M}$; and SB203580, $10 \mu \mathrm{M}$ ). The statistical results are presented in a column diagram. Data are represented as the mean \pm standard error of the mean from three independent experiments. "P $<0.05$ and ${ }^{* *} \mathrm{P}<0.01$ indicate a significant alteration in the expression levels of proteins after the treatment with MAPK inhibitors. The expression of GAPDH was used as an internal control. All immunoblotting experiments were performed in duplicates. Bcl, B-cell lymphoma; Bax, Bcl-2-associated X protein; xL, extra large; Mcl-1, myeloid cell leukemia-1; Bim, Bcl-2-like 11; HP, Huaier polysaccharide; p, phosphorylated; GAPDH, glyceraldehyde 3-phosphate dehydrogenase; MAPK, mitogen-activated protein kinase.

illustrated in Fig. 4A, the HP-activated levels of ERK1/2, JNK and p38 in HepG2 and Huh7 cells were blocked markedly in the presence of the corresponding inhibitor at its specific concentration $(20 \mu \mathrm{M}$ for PD98059, $10 \mu \mathrm{M}$ for SP600125 and $10 \mu \mathrm{M}$ for SB203580). Fig. 4B displays the decreased HP-induced growth inhibition in HepG2 and Huh7 cells by SB203580 treatment, while the other inhibitors had no significant effects. Flow cytometry analysis demonstrated that the HP-triggered apoptosis in HepG2 and Huh7 cells was attenuated by SB203580 (Fig. 4C). Notably, the application of SP600125 led to decreased apoptosis in HP-treated HepG2 cells but not in Huh7 cells.

Inactivation of 338 MAPK modulates the effect of HP on the expression of apoptosis-related proteins. To explore the underlying mechanism by which the inactivation of $\mathrm{p} 38$ 
MAPK pathway attenuates the apoptosis induced by HP, the expression levels of a variety of apoptosis-related proteins were investigated. As shown in Fig. 5A, the treatment with HP increased the expression of the pro-apoptotic proteins Bim, Bax and p53 in HepG2 and Huh7 cells in a time-dependent manner, while the expression of the pro-survival proteins Bcl-2, Bcl-xL, Mcl-1 and survivin was downregulated. Next, HepG2 and Huh7 cells were treated with HP for $24 \mathrm{~h}$ in the presence or absence of the aforementioned MAPK inhibitors. Fig. 5B indicates that SB203580 treatment significantly increased the expression of Bcl-2 and survivin in HP-treated HepG2 cells compared with cells subjected to treatment with HP alone, whereas the expression of Bax was substantially downregulated by the combination treatment. Similar results were obtained in Huh7 cells (Fig. 5C). However, the expression levels of Bax, Bcl-2 and survivin were all increased in HepG2 and Huh7 cells following the combination treatment with SP600125 and HP, compared with the application of HP alone (Fig. 5B and C). Notably, treatment with PD98059 considerably augmented the expression of Bax in HP-treated HepG2 cells in comparison with its expression levels when only HP was utilized (Fig. 5B), while the expression levels of survivin were decreased and those of $\mathrm{Bcl}-2$ remained unchanged. In the HP-treated Huh7 cells, the expression of survivin was significantly reduced in the presence of PD98059, which was accompanied by a decline in the expression levels of $\mathrm{Bcl}-2$ and an unchanged level exhibited by Bax (Fig. 5C).

\section{Discussion}

In the present study, the role of the MAPK signaling pathways in HP-induced apoptosis in HCC cells was investigated. It was observed that HP activates the three major MAPK signaling pathways, namely ERK, JNK and p38 MAPK, in HepG2 and Huh7 HCC cells. Furthermore, the present findings provide evidence that the pharmacological inhibition of p38 MAPK attenuates HP-induced apoptosis in both cell lines. The present data demonstrate for the first time a role of p38 MAPK in the HP-mediated effect on HCC cells.

Recent studies have established that Huaier or HP manifest antitumor effects in a variety of cancer cells in vitro and in vivo, and various signaling pathways are involved in the Huaier or HP-triggered antitumor activities in different types of cancer cells. For instance, Huaier suppressed the growth of colorectal cancer stem cells partially via downregulation of the Wnt/ $\beta$-catenin signaling pathway (26), whereas the upregulation of microRNA-26b-5p contributed to the Huaier-induced apoptosis in human pulmonary cancer cells (15). In addition, Huaier inhibited ovarian cancer cell motility via the AKT/glycogen synthase kinase $3 \beta / \beta$-catenin signaling pathway (16). HP exhibited prominent antitumor activities in vivo via enhancement of the host immune system function in HCC tumor-bearing mice (17). Additionally, HP was able to suppress human HCC MHCC97-H cell metastasis via inactivation of the epithelial-mesenchymal transition and the astrocyte elevated gene-1 signaling pathway (12).

The MAPK signaling pathways are generally subdivided into three separate pathways: ERK, JNK and p38 MAPK (20,27). Normally, p38 MAPK activities are significantly lower in $\mathrm{HCC}$, while the activation of p38 MAPK may cause apoptosis of HCC cells (28). Previous studies have demonstrated that certain toxic agents induce cell apoptosis via activation of p38 MAPK (29). The present findings revealed that HP induced cell cycle arrest and apoptosis, and activated the three major MAPK pathways in HCC cells, although the expression of p38 MAPK significantly increased compared with that of JNK and ERK. However, upon treatment with the specific MAPK inhibitors PD98059, SP600125 and SB203580 (targeting MEK, JNK and p38 MAPK, respectively), it was observed that the inactivation of p38 MAPK markedly antagonized the effect of HP-induced apoptosis and inhibition of proliferation in both HepG2 and Huh7 cells, indicating that p38 MAPK may play a role in the process of apoptosis. The present results also revealed that HP diminished the phosphorylation levels of AKT and mTOR, which is consistent with previous findings reported for HP-treated ovarian cancer cells and MHCC97-H cells $(13,16)$.

The present study further unveiled the potential molecular mechanisms by which p38 MAPK contributes to HP-induced apoptosis in HCC cells. It has been reported that p38 MAPK acts as a tumour suppressor in various cancer cells (30), and activated p38 MAPK is able to induce apoptosis by regulating several proteins, including p53 and Bcl-2 (31-33). Several studies have indicated that Huaier and HP modulate the expression of apoptosis-related proteins, including p53, Bcl-2, Bcl-xL and Bax (11,19,34), which may be a critical mechanism employed by HP to mediate its effect on cancer cell death (35). The present data support the above observations, since upon treatment with HP, the expression of the pro-survival proteins Bcl-2 and Bcl-xL decreased, whereas that of the pro-apoptotic proteins Bax and p53 increased, thereby promoting the HP-induced apoptosis. In addition, the present study is the first to report that HP downregulates the expression of survivin in HCC cells, which is an inhibitor of apoptotic proteins $(36,37)$. Importantly, it was observed that the inactivation of p38 MAPK, but not that of JNK or ERK, simultaneously antagonized the effect of HP on the expression of the apoptosis-related proteins $\mathrm{Bcl}-2$, Bax and survivin, thereby suggesting a potential mechanism by which p38 MAPK contributes to the HP-induced apoptosis.

Previous in vivo data demonstrated that p53 expression was downregulated in HCC mouse models $(11,19)$. However, the present findings are contradictory to these observations, as increased expression of p53 was observed in HepG2 cells expressing wild-type and functional p53, and in Huh7 cells carrying the mutated and inactivated p53 gene (23). The results of the current study suggested that HP could induce apoptosis in HCC cells regardless of the p53 status. Based on the activity of p53 as a tumor suppressor in cancer, its role in HP-induced apoptosis requires to be further investigated.

In summary, the present study provides evidence that HP induces apoptosis in HCC cells expressing either the wild-type or the mutated p53 gene. Furthermore, it demonstrates the role of p38 MAPK in HP-triggered cancer cell death. Thus, the present findings enhance the understanding and provide novel insights into the underlying mechanism by which HP exerts its antitumor effect. 


\section{Acknowledgements}

The authors would like to thank Dr Songshu Meng (Institute of Cancer Stem Cell, Dalian Medical University Cancer Center, Dalian, China), for providing guidance in the scientific research and critical reading of the manuscript. The present study was partly supported by the Specialized Research Fund for the Doctoral Program of Higher Education (Beijing, China; grant no. 20122105110009), the National Natural Science Foundation of China (Beijing, China; grant no. 81473504), the Program for Excellent Talents in Universities of Liaoning province (Liaoning, China; grant no. LR201518), the Hundred, Thousand, and Ten Thousand Talent Project of Liaoning province (Liaoning, China; grant no. 2015-234) and the Distinguished Professor Award of Liaoning Province (Liaoning, China; grant no. 2013-204).

\section{References}

1. Torre LA, Bray F, Siegel RL, Ferlay J, Lortet-Tieulent J and Jemal A: Global cancer statistics, 2012. CA Cancer J Clin 65 87-108, 2015

2. Bosch FX, Ribes J, Diaz M and Cléries R: Primary liver cancer: Worldwide incidence and trends. Gastroenterology 127 (5 Suppl 1): S5-S16, 2004.

3. Ferenci P, Fried M, Labrecque D, Bruix J, Sherman M, Omata M, Heathcote J, Piratsivuth T, Kew M, Otegbayo JA, et al: World gastroenterology organisation guideline. Hepatocellular carcinoma (HCC): A global perspective. J Gastrointestin Liver Dis 19: 311-317, 2010.

4. Thomas MB and Abbruzzese JL: Opportunities for targeted therapies in hepatocellular carcinoma. J Clin Oncol 23: 8093-8108, 2005.

5. Signoriello S, Annunziata A, Lama N, Signoriello G, Chiodini P, De Sio I, Daniele B, Di Costanzo GG, Calise F, Olivieri G, et al: Survival after locoregional treatments for hepatocellular carcinoma: A cohort study in real-world patients. Scientific World Journal 2012: 564706, 2012.

6. Chan SL and Yeo W: Targeted therapy of hepatocellular carcinoma: Present and future. J Gastroenterol Hepatol 27: 862-872, 2012.

7. Lu SC: Where are we in the chemoprevention of hepatocellular carcinoma? Hepatology 51: 734-736, 2010.

8. Chen XP, He SQ, Zhao X, Huang ZY and Li CH: Chinese medicine Extractum trametes robiniophila murr augment tumor necrosis factor related apoptosis-inducing ligand induced apoptosis in human hepatic cancer cell lines. Zhonghua Wai Ke Za Zhi 43: 1524-1527, 2005 (In Chinese).

9. Zhang N, Kong X, Yan S, Yuan C and Yang Q: Huaier aqueous extract inhibits proliferation of breast cancer cells by inducing apoptosis. Cancer Sci 101: 2375-2383, 2010.

10. Wang X, Zhang N, Huo Q, Sun M, Dong L, Zhang Y, Xu G and Yang Q: Huaier aqueous extract inhibits stem-like characteristics of MCF7 breast cancer cells via inactivation of hedgehog pathway. Tumour Biol 35: 10805-10813, 2014.

11. Ren J, Zheng C, Feng G, Liang H, Xia X, Fang J, Duan X and Zhao $\mathrm{H}$ : Inhibitory effect of extract of fungi of Huaier on hepatocellular carcinoma cells. J Huazhong Univ Sci Technolog Med Sci 29: 198-201, 2009.

12. Zheng J, Li C, Wu X, Liu M, Sun X, Yang Y, Hao M, Sheng S, Sun Y, Zhang H, et al: Huaier polysaccharides suppresses hepatocarcinoma MHCC97-H cell metastasis via inactivation of EMT and AEG-1 pathway. Int J Biol Macromol 64: 106-110, 2014.

13. Zheng J, Li C, Wu X, Liu M, Sun X, Yang Y, Hao M, Sheng S, Sun Y,Zhang H, et al: Astrocyte elevated gene-1 (AEG-1) shRNA sensitizes Huaier polysaccharide (HP)-induced anti-metastatic potency via inactivating downstream P13K/Akt pathway as well as augmenting cell-mediated immune response. Tumour Biol 35: 4219-4224, 2014.

14. Li C, Wu X, Zhang H, Yang G, Hao M, Sheng S, Sun Y, Long J, $\mathrm{Hu} \mathrm{C}$, Sun X, et al: A Huaier polysaccharide inhibits hepatocellular carcinoma growth and metastasis. Tumour Biol 36: 1739-1745, 2015.
15. Wu T, Chen W, Liu S, Lu H, Wang H, Kong D, Huang X, Kong Q, Ning Y and Lu Z: Huaier suppresses proliferation and induces apoptosis in human pulmonary cancer cells via upregulation of miR-26b-5p. FEBS Lett 588: 2107-2114, 2014.

16. Yan X, Lyu T, Jia N, Yu Y, Hua K and Feng W: Huaier aqueous extract inhibits ovarian cancer cell motility via the AKT/GSK3 $\beta /$ $\beta$-catenin pathway. PLoS One 8: e63731, 2013.

17. Li C, Wu X, Zhang H, Yang G, Hao M, Sheng S, Sun Y, Long J, $\mathrm{Hu} \mathrm{C}$, Sun X, et al: A Huaier polysaccharide restrains hepatocellular carcinoma growth and metastasis by suppression angiogenesis. Int J Biol Macromol 75: 115-120, 2015.

18. Wang X, Zhang N, Huo Q and Yang Q: Anti-angiogenic and antitumor activities of Huaier aqueous extract. Oncol Rep 28: $1167-1175,2012$

19. Xu X, Wei Q, Wang K, Ling Q, Xie H, Zhou L and Zheng S: Anticancer effects of Huaier are associated with down-regulation of P53. Asian Pac J Cancer Prev 12: 2251-2254, 2011.

20. Chang L and Karin M: Mammalian MAP kinase signalling cascades. Nature 410: 37-40, 2001

21. Burotto M, Chiou VL, Lee JM and Kohn EC: The MAPK pathway across different malignancies: A new perspective. Cancer 120: 3446-3456, 2014.

22. Haakenson J, Wu JY, Xiang S, Williams KA, Bai W and Zhang X: HDAC6-Dependent functions in tumor cells: Crossroad with the MAPK Pathways. Crit Rev Oncog 20: 65-81, 2015.

23. Luo Z, Yu G, Lee HW, Li L, Wang L, Yang D, Pan Y, Ding C, Qian J, Wu L, et al: The Nedd8-activating enzyme inhibitor MLN4924 induces autophagy and apoptosis to suppress liver cancer cell growth. Cancer Res 72: 3360-3371, 2012.

24. Casimiro MC, Velasco-Velázquez M, Aguirre-Alvarado C and Pestell RG: Overview of cyclins D1 function in cancer and the CDK inhibitor landscape: Past and present. Expert Opin Investig Drugs 23: 295-304, 2014

25. Pestell RG: New roles of cyclin D1. Am J Pathol 183: 3-9, 2013.

26. Rathmell JC and Thompson CB: The central effectors of cell death in the immune system. Annu Rev Immunol 17: 781-828, 1999.

27. Hsieh SC, Huang MH, Cheng CW, Hung JH, Yang SF and Hsieh YH: $\alpha$-Mangostin induces mitochondrial dependent apoptosis in human hepatoma SK-Hep-1 cells through inhibition of p38 MAPK pathway. Apoptosis 18: 1548-1560, 2013.

28. Lamy E, Herz C, Lutz-Bonengel S, Hertrampf A, Márton MR and Mersch-Sundermann V: The MAPK pathway signals telomerase modulation in response to isothiocyanate-induced DNA damage of human liver cancer cells. PLoS One 8: e53240, 2013.

29. Chiba T, Suzuki E, Yuki K, Zen Y, Oshima M, Miyagi S, Saraya A, Koide S, Motoyama T, Ogasawara S, et al: Disulfiram eradicates tumor-initiating hepatocellular carcinoma cells in ROS-p38 MAPK pathway-dependent and -independent manners. PLoS One 9: e84807, 2014.

30. Jiang Q, Li F, Shi K, Wu P, An J, Yang Y and Xu C: ATF4 activation by the p38MAPK-eIF4E axis mediates apoptosis and autophagy induced by selenite in Jurkat cells. FEBS Lett 587: 2420-2429, 2013.

31. Taylor CA, Zheng Q, Liu Z and Thompson JE: Role of p38 and JNK MAPK signaling pathways and tumor suppressor p53 on induction of apoptosis in response to Ad-eIF5A1 in A549 lung cancer cells. Mol Cancer 12: 35, 2013.

32. Hui K, Yang Y, Shi K, Luo H, Duan J, An J, Wu P, Ci Y, Shi L and $\mathrm{Xu}$ C: The p38 MAPK-regulated PKD1/CREB/Bcl-2 pathway contributes to selenite-induced colorectal cancer cell apoptosis in vitro and in vivo. Cancer Lett 354: 189-199, 2014.

33. Zhang X, Bi L, Ye Y and Chen J: Formononetin induces apoptosis in PC-3 prostate cancer cells through enhancing the Bax/Bcl-2 ratios and regulating the p38/Akt pathway. Nutr Cancer 66: 656-661, 2014.

34. Hengartner MO: The biochemistry of apoptosis. Nature 407: 770-776, 2000

35. Mayo LD and Donner DB: The PTEN, Mdm2, p53 tumor suppressor-oncoprotein network. Trends Biochem Sci 27: 462-467, 2002.

36. Li F, Ambrosini G, Chu EY, Plescia J, Tognin S, Marchisio PC and Altieri DC: Control of apoptosis and mitotic spindle checkpoint by survivin. Nature 396: 580-584, 1998.

37. Fernández JG, Rodriguez DA, Valenzuela M, Calderon C, Urzúa U, Munroe D, Rosas C, Lemus D, Díaz N, Wright MC, et al: Survivin expression promotes VEGF-induced tumor angiogenesis via PI3K/Akt enhanced $\beta$-catenin/Tcf-Lef dependent transcription. Mol Cancer 13: 209, 2014. 